\title{
On the Stream Function-Vorticity Finite Element Formulation for Incompressible Flow in Porous Media
}

\author{
Abdellatif Agouzal, ${ }^{1}$ Karam Allali, ${ }^{2}$ and Siham Binna ${ }^{2}$ \\ ${ }^{1}$ University Lyon 1, CNRS UMR 5208, Institute Camille Jordan, 69100 Villeurbanne, France \\ ${ }^{2}$ University Hassan II, FSTM, P.O. Box 146, Mohammedia, Morocco \\ Correspondence should be addressed to Karam Allali; allali@hotmail.com
}

Received 28 May 2014; Revised 30 August 2014; Accepted 3 September 2014; Published 18 September 2014

Academic Editor: Zhangxin Chen

Copyright (C) 2014 Abdellatif Agouzal et al. This is an open access article distributed under the Creative Commons Attribution License, which permits unrestricted use, distribution, and reproduction in any medium, provided the original work is properly cited.

Stream function-vorticity finite element formulation for incompressible flow in porous media is presented. The model consists of the heat equation, the equation for the concentration, and the equations of motion under the Darcy law. The existence of solution for the discrete problem is established. Optimal a priori error estimates are given.

\section{Introduction}

The stream function-vorticity formulation is widely used to perform the numerical simulation of an incompressible fluid flow in porous media $[1,2]$. The main advantage of the formulation is a reduction of the numerical problem unknowns with the fact that the continuity equation remains satisfied. In this paper, we are interested in studying the finite element of stream function-vorticity formulation for propagating reaction front in porous media. The model considered is a system of reaction-diffusion equations coupled with the hydrodynamic under the Darcy-Boussinesq approximation in the open bounded convex domain $\Omega \subset \mathbb{R}^{2}$ [3-5]:

$$
(P)\left\{\begin{array}{l}
\partial_{t} T-\lambda \Delta T+u \cdot \nabla T=\alpha C \exp \left(-\frac{E}{R T}\right), \\
\partial_{t} C-\eta \Delta C+u \cdot \nabla C=-\alpha C \exp \left(-\frac{E}{R T}\right), \\
\partial_{t} u+\frac{\mu}{K} u+\nabla p=\beta\left(T-T_{0}\right) g \gamma, \\
\operatorname{div} u=0,
\end{array}\right.
$$

where $T$ is the temperature, $C$ is the concentration, $u$ is the velocity, $p$ is the pressure, $\lambda$ is the thermal diffusivity, $\eta$ is the diffusion, $\mu$ is the viscosity, $K$ is the permeability, $E$ is the activation energy, $R$ is the universal gas constant, $\alpha$ is the Arrhenius preexponential factor, $T_{0}$ is a mean value of temperature, $g$ is the gravity, $\gamma$ is the upward unit vector, and $\beta$ is the coefficient of the thermal expansion of the fluid.

The boundary conditions are of Dirichlet-Neumann type for the temperature and the concentration and of impermeability type for the normal component of velocity:

$$
\begin{gathered}
\left.T\right|_{\Gamma_{1}}=\left.C\right|_{\Gamma_{1}}=0,\left.\quad \frac{\partial T}{\partial n}\right|_{\Gamma_{2}}=\left.\frac{\partial C}{\partial n}\right|_{\Gamma_{2}}=0, \\
\left.u \cdot n\right|_{\partial \Omega}=0, \\
\left.u\right|_{t=0}=u_{0},\left.\quad T\right|_{t=0}=T_{0},\left.\quad C\right|_{t=0}=C_{0},
\end{gathered}
$$

where $\Gamma_{1}$ and $\Gamma_{2}$ are disjoint open parts of $\partial \Omega$ such that $\overline{\Gamma_{1}} \cup$ $\overline{\Gamma_{2}}=\partial \Omega$.

Due to the incompressibility of the fluid, we introduce the stream function $\psi$ :

$$
u=\left(\begin{array}{c}
\frac{\partial \psi}{\partial y} \\
-\frac{\partial \psi}{\partial x}
\end{array}\right)
$$


and by introducing also the vorticity $\omega=\operatorname{curl}(u)$, the problem $(P)$ becomes

$$
(\mathscr{P})\left\{\begin{array}{l}
\partial_{t} T-\lambda \Delta T+\operatorname{rot}(\psi) \cdot \nabla T-C g(T)=0, \\
\partial_{t} C-\eta \Delta C+\operatorname{rot}(\psi) \cdot \nabla C+C g(T)=0, \\
\partial_{t} \omega+\mu_{p} \omega=f(T), \\
-\Delta \psi=\omega,
\end{array}\right.
$$

where $g(T)=\alpha \exp (-E / R T), f(T)=\beta g(\partial T / \partial x)$, and $\mu_{p}=$ $\mu / K$.

Because there is no flow of fluid through the boundary (impermeability boundary), we will have the following zeroflux condition:

$$
\left.\psi\right|_{\partial \Omega}=0
$$

The paper is organized as follows. We present the variational formulation of the problem in the next section. We establish the existence result in Section 3. A priori error estimates are given in Section 4. We conclude in the last section.

\section{The Variational Formulation}

In order to state the problem in the variational form, we first describe the functional framework needed for our study. We set

$$
\begin{gathered}
X=L^{2}\left(0, t, H_{0}^{1}(\Omega)\right), \quad W=L^{2}\left(0, t, H_{0, \Gamma_{1}}^{1}(\Omega)\right), \\
M=L^{2}\left(0, t, L^{2}(\Omega)\right) .
\end{gathered}
$$

We introduce now the constant of Friedrichs-Poincaré which depends on the geometry of the domain $\Omega$ :

$$
\rho=\sup _{u \in H_{0}^{1}(\Omega)} \frac{\|u\|_{L^{2}(\Omega)}}{\|\nabla u\|_{L^{2}(\Omega)}} .
$$

The variational form of the continued problem $(\mathscr{P})$ can be written as the following form:

$$
\left(\mathscr{P}_{v}\right)\left\{\begin{array}{c}
\text { Find a }(\psi, \omega, T, C) \in X \times \bar{M} \times \bar{W}^{2} \text {, such as, } \\
\forall(\zeta, v, \phi, \xi) \in X \times M \times W^{2}, \text { we have: } \\
\left(\partial_{t} T, \phi\right)+\lambda(\nabla T, \nabla \phi)+\frac{1}{2} \int_{\Omega}(\operatorname{rot}(\psi) \nabla T) \phi \\
-\frac{1}{2} \int_{\Omega}(\operatorname{rot}(\psi) \nabla \phi) T=\int_{\Omega} C g(T) \phi, \\
\left(\partial_{t} C, \xi\right)+\eta(\nabla C, \nabla \xi)+\frac{1}{2} \int_{\Omega}(\operatorname{rot}(\psi) \nabla C) \xi \\
-\frac{1}{2} \int_{\Omega}(\operatorname{rot}(\psi) \nabla \xi) C=-\int_{\Omega} C g(T) \xi, \\
\left(\partial_{t} \omega, v\right)+\mu_{p}(\omega, v)=\int_{\Omega} f(T) v, \\
(\nabla \psi, \nabla \zeta)=(\omega, \zeta),
\end{array}\right.
$$

where

$$
\begin{gathered}
\bar{M}=\left\{\omega \in M, \frac{\partial \omega}{\partial t} \in L^{2}\left(0, t, L^{2}(\Omega)\right)\right\}, \\
\bar{W}=\left\{T \in W, \frac{\partial T}{\partial t} \in L^{2}\left(0, t,\left(H_{0, \Gamma_{1}}^{1}(\Omega)\right)^{*}\right)\right\} .
\end{gathered}
$$

Here, $\left(H_{0, \Gamma_{1}}^{1}(\Omega)\right)^{*}$ is the dual of the space $H_{0, \Gamma_{1}}^{1}(\Omega)$. We can see clearly that the parameters and the functions $f$ and $g$ of the problem verify the following conditions:

The reals $\mu_{p}, \eta$, and $\lambda$ are strictly positives,

$$
\begin{gathered}
g \in W^{1, \infty}(\mathbb{R}), \quad\left\|g^{\prime}\right\|_{L^{\infty}(\Omega)}=\frac{E \alpha}{R T_{i}^{2}}, \quad g \geq 0, \\
\|g\|_{L^{\infty}(\Omega)}=\alpha, \\
f \in W^{1, \infty}(\mathbb{R}), \quad f\left(T_{0}\right)=0, \\
\forall\left(T_{1}, T_{2}\right) \in\left(H_{0, \Gamma_{1}}^{1}(\Omega)\right)^{2}, \\
\left\|f\left(T_{1}\right)-f\left(T_{2}\right)\right\|_{L^{2}(\Omega)} \leq \beta g\left\|\nabla\left(T_{1}-T_{2}\right)\right\|_{L^{2}(\Omega)},
\end{gathered}
$$

where $T_{i}$ is the temperature of the unburned mixture. For convenience, for all $(u, v) \in X^{2}$ and $(T, \phi, C, \psi) \in W^{4}$, we introduce the forms defined by

$$
\begin{aligned}
a(u, v) & =\left(\int_{\Omega} \nabla u \nabla v d x\right), \\
a_{1}(u, T, \phi) & \frac{1}{2}\left(\int_{\Omega}(\operatorname{rot}(u) \nabla) T \phi d x-\int_{\Omega}(\operatorname{rot}(u) \nabla) \phi T d x\right), \\
j(T, \psi) & =\int_{\Omega} \nabla T \nabla \psi d x, \\
k(C, T, \psi) & =\int_{\Omega} C g(T) \psi d x .
\end{aligned}
$$

The variational formulation $\left(\mathscr{P}_{v}\right)$ is rewritten as follows:

$$
\left(\mathscr{P}_{v}\right)\left\{\begin{array}{c}
\text { Find } \mathrm{a}(\psi, \omega, T, C) \in X \times \bar{M} \times \bar{W}^{2}, \text { such as, } \\
\quad \forall(v, u, \phi, \xi) \in M \times X \times W^{2}, \text { we have: } \\
\left(\partial_{t} T, \phi\right)+\lambda j(T, \phi)+a_{1}(\psi, T, \phi) \\
-k(C, T, \phi)=0, \quad \forall \phi \in W, \\
\left(\partial_{t} C, \xi\right)+\eta j(C, \xi)+a_{1}(\psi, C, \xi) \\
\quad+k(C, T, \xi)=0, \quad \forall \xi \in W, \\
\left(\partial_{t} \omega, v\right)+\mu_{p}(\omega, v)=(f(T), v), \quad \forall v \in M, \\
a(\psi, u)=(\omega, u), \quad \forall u \in X .
\end{array}\right.
$$




\section{The Existence Result}

3.1. The Semidiscrete Problem. In order to give the semidiscrete problem, we will need the following spaces:

$$
X_{h} \subset H_{0}^{1}(\Omega), \quad M_{h} \subset L^{2}(\Omega), \quad W_{h} \subset H_{0, \Gamma_{1}}^{1}(\Omega),
$$

where $h$ is a strictly positive constant. We assume that the spaces $X_{h}, M_{h}$, and $W_{h}$ satisfy the following assumptions.

(1) For all $0<\sigma \leq 1$, there exists a linear continuous operator $R_{h}$ from $W^{1+\sigma, 4}(\Omega) \cap \stackrel{\circ}{W}^{1,4}(\Omega)$ onto $X_{h}$ such that, for all $\psi \in W^{1+\sigma, 4}(\Omega) \cap \stackrel{\circ}{W}^{1,4}(\Omega)$,

$$
\begin{gathered}
\left\|\psi-R_{h} \psi\right\|_{1,4} \leqslant h^{\sigma}|\psi|_{1+\sigma, 4}, \\
\int_{\Omega} \nabla\left(\psi-R_{h} \psi\right) \nabla v_{h}=0 \quad \forall v_{h} \in X_{h} .
\end{gathered}
$$

(2) For all $0<\sigma \leq 1$, there exists a linear continuous operator $i_{h}$ from $H^{1+\sigma}(\Omega) \cap H_{0}^{1}(\Omega)$ onto $W_{h}$ such that, for all $T \in H^{1+\sigma}(\Omega) \cap H_{0}^{1}(\Omega)$,

$$
\begin{gathered}
\left\|T-i_{h} T\right\|_{1, \Omega} \leq h^{\sigma}|T|_{1+\sigma, \Omega}, \\
\int_{\Omega} \nabla\left(T-i_{h} T\right) \nabla v_{h}=0 \quad \forall v_{h} \in X_{h} .
\end{gathered}
$$

Examples of such spaces verifying these conditions are given in $[6,7]$.

The discretized form of the problem $\left(\mathscr{P}_{v}\right)$ is given as follows:

$$
\left(\mathscr{P}_{h}\right)\left\{\begin{array}{c}
\text { Find } \omega_{h} \in C^{1}\left(0, t, M_{h}\right), \psi_{h} \in C^{0}\left(0, t, X_{h}\right), \\
\text { and }\left(C_{h}, T_{h}\right) \in\left(C^{1}\left(0, t, W_{h}\right)\right)^{2} \text { such that: } \\
\left(\partial_{t} T_{h}, \phi_{h}\right)+\lambda j\left(T_{h}, \phi_{h}\right)+a_{1}\left(\psi_{h}, T_{h}, \phi_{h}\right) \\
-k\left(C_{h}, T_{h}, \phi_{h}\right)=0, \quad \forall \phi_{h} \in W_{h}, \\
\quad+k\left(C_{h}, T_{h}, \xi_{h}\right)=0, \quad \forall \xi_{h} \in W_{h}, \\
\left(\partial_{t} \omega_{h}, v_{h}\right)+\mu_{p}\left(\omega_{h}, v_{h}\right)=\left(f\left(T_{h}\right), v_{h}\right), \\
\forall v_{h} \in M_{h}, \\
a\left(\psi_{h}, u_{h}\right)=\left(\omega_{h}, u_{h}\right), \quad \forall u_{h} \in X_{h} .
\end{array}\right.
$$

With the initial conditions,

$$
\begin{gathered}
\omega_{h}(t=0)=\omega_{h}^{0} \in M_{h}, \quad T_{h}(t=0)=T_{h}^{0} \in W_{h}, \\
C_{h}(t=0)=C_{h}^{0} \in W_{h} .
\end{gathered}
$$

In the sequel, we assume for simplicity that

$$
\omega_{0}=\omega_{h}^{0}, \quad T_{0}=T_{h}^{0}, \quad C_{0}=C_{h}^{0} .
$$

The main result of the paper is written as follows.

Theorem 1. The problem $\left(\mathscr{P}_{h}\right)$ admits a unique solution. Moreover, for $\psi, \omega, T$, and $C$ solutions of the problem $\left(\mathscr{P}_{v}\right)$ and for $\psi_{h}, \omega_{h}, T_{h}$, and $C_{h}$ solutions of the problem $\left(\mathscr{P}_{h}\right)$, one has

$$
\begin{aligned}
& \left\|\psi-\psi_{h}\right\|_{L^{2}\left(0, t,\left(W^{1,4}(\Omega)\right)\right)} \\
& +\left\|T-T_{h}\right\|_{L^{2}\left(0, t, H_{0, I_{1}}^{1}(\Omega)\right)} \\
& +\left\|C-C_{h}\right\|_{L^{2}\left(0, t, H_{0, I_{1}}^{1}(\Omega)\right)} \\
& \quad+\left\|\omega-\omega_{h}\right\|_{L^{2}\left(0, t, L^{2}(\Omega)\right)} \\
& \leq h^{\sigma}\left(\|T\|_{L^{2}\left(0, t, H^{1+\sigma}(\Omega)\right)}+\|T\|_{H^{1}\left(0, t, H^{\sigma}(\Omega)\right)}\right. \\
& \quad+\|C\|_{L^{2}\left(0, t, H^{1+\sigma}(\Omega)\right)}+\|C\|_{H^{1}\left(0, t, H^{\sigma}(\Omega)\right)} \\
& \quad+\|\omega\|_{L^{2}\left(0, t, H^{\sigma}(\Omega)\right)}+\|\omega\|_{H^{1}\left(0, t, H^{\sigma}(\Omega)\right)} \\
& \left.\quad+\|\psi\|_{L^{2}\left(0, t, W^{1+\sigma, 4}(\Omega)\right)}\right),
\end{aligned}
$$

under the following conditions:

$$
\begin{gathered}
\delta_{1}=1-\frac{\sqrt{5} N_{C} \alpha E \rho^{2}}{R T_{b}^{2} \lambda}>0, \\
\delta_{2}=1-\frac{2 \sqrt{5} \rho^{4} \alpha^{2} E N_{C}}{\delta_{1} \lambda \eta R T_{b}^{2}}>0, \\
\delta_{2} \delta_{1}>\frac{2 \sqrt{3} \beta g N}{\lambda \mu_{p}}\left(\frac{2 \sqrt{10} \alpha \rho^{2} N_{C}}{\eta}+\sqrt{5} N_{T}\right),
\end{gathered}
$$

where

$$
\begin{gathered}
N_{T}=\sup \left(\left\|T_{h}\right\|_{L^{\infty}\left(0, t, H_{0, I_{1}}^{1}(\Omega)\right)},\|T\|_{L^{\infty}\left(0, t, H_{0, \Gamma_{1}}^{1}(\Omega)\right)}\right), \\
N_{C}=\sup \left(\left\|C_{h}\right\|_{L^{\infty}\left(0, t, H_{0, I_{1}}^{1}(\Omega)\right)}\|C\|_{L^{\infty}\left(0, t, H_{0, \Gamma_{1}}^{1}(\Omega)\right)}\right), \\
M_{1}=\sup \left(\left\|\psi_{h}\right\|_{L^{\infty}\left(0, t,\left(W^{1,4}(\Omega)\right)\right)}\|\psi\|_{L^{\infty}\left(0, t,\left(W^{1,4}(\Omega)\right)\right)}\right), \\
N=\sup _{\psi \in \stackrel{W}{ }(\Omega), v \in\left(H_{0}^{1}\right)(\Omega), w \in\left(H_{0}^{1}\right)(\Omega)} \frac{a_{1}(\psi, v, w)}{\|v\|_{H_{0}^{1}(\Omega)}\|\psi\|_{\dot{W}^{1,4}(\Omega)}\|w\|_{H_{0}^{1}(\Omega)}}, \\
0<\sigma \leq 1 .
\end{gathered}
$$

Throughout the paper, we often use the following notation.

For each $\zeta, \eta>0: \zeta \lesssim \eta \Leftrightarrow \exists C^{\star}>0: \zeta \leq C^{\star} \eta$; without further specification, the constant $C^{\star}$ is independent of the mesh size and the solutions. 
3.2. Existence of Semidiscrete Solutions. In order to prove the existence result of the problem $\left(\mathscr{P}_{h}\right)$, we need the following lemmas; first for the concentration, we have the following.

Lemma 2. For any local solution $C_{h}$ of the problem $\left(\mathscr{P}_{h}\right)$, one has the a priori estimate

$$
\left\|C_{h}\right\|_{L^{\infty}\left(0, t, L^{2}(\Omega)\right)}^{2}+2 \eta\left\|C_{h}\right\|_{L^{2}\left(0, t, H_{0, \Gamma_{1}}^{1}(\Omega)\right)}^{2} \leq 2\left\|C_{h}^{0}\right\|_{L^{2}(\Omega)}^{2} .
$$

Proof. By choosing $\xi_{h}=C_{h}$, as test function in the third equation of the problem $\left(\mathscr{P}_{h}\right)$, we have

$$
\frac{1}{2} \frac{d}{d t}\left\|C_{h}\right\|_{L^{2}(\Omega)}^{2}+\eta\left\|\nabla C_{h}\right\|_{L^{2}(\Omega)}^{2}+a_{1}\left(C_{h}, T_{h}, C_{h}\right)=0 .
$$

Integrating the last equality and noticing that $a_{1}\left(C_{h}, T_{h}, C_{h}\right)$ is positive, we obtain

$$
\begin{gathered}
2 \eta\left\|C_{h}\right\|_{L^{2}\left(0, t, H_{0, \Gamma_{1}}^{1}(\Omega)\right)}^{2} \leq\left\|C_{h}^{0}\right\|_{L^{2}(\Omega)}^{2}, \\
\left\|C_{h}(t=s)\right\|_{L^{2}(\Omega)}^{2} \leq\left\|C_{h}^{0}\right\|_{L^{2}(\Omega)}^{2} .
\end{gathered}
$$

By using the two last inequalities, we obtain the result of the lemma.

Also, for the temperature, we have the following result.

Lemma 3. For any local solution $T_{h}$ of the problem $\left(\mathscr{P}_{h}\right)$, one has the a priori estimate

$$
\begin{gathered}
\left\|T_{h}\right\|_{L^{\infty}\left(0, t, L^{2}(\Omega)\right)}^{2}+\lambda\left\|T_{h}\right\|_{L^{2}\left(0, t, H_{0, \Gamma_{1}}^{1}(\Omega)\right)}^{2} \\
\quad \leq \frac{(\alpha \rho)^{2}}{\lambda \eta}\left\|C_{h}^{0}\right\|_{L^{2}(\Omega)}^{2}+2\left\|T_{h}^{0}\right\|_{L^{2}(\Omega)}^{2} .
\end{gathered}
$$

Proof. By choosing $\phi_{h}=T_{h}$, as test function in the second equation of the problem $\left(\mathscr{P}_{h}\right)$, we have

$$
\begin{aligned}
\frac{1}{2} \frac{d}{d t} \| & T_{h}\left\|_{L^{2}(\Omega)}^{2}+\lambda\right\| \nabla T_{h} \|_{L^{2}(\Omega)}^{2} \\
& =k\left(C_{h}, T_{h}, T_{h}\right) \\
& \leq \rho \alpha\left\|C_{h}\right\|_{L^{2}(\Omega)}\left\|\nabla T_{h}\right\|_{L^{2}(\Omega)} \\
& \leq \frac{(\alpha \rho)^{2}}{2 \lambda}\left\|C_{h}\right\|_{L^{2}(\Omega)}^{2}+\frac{\lambda}{2}\left\|\nabla T_{h}\right\|_{L^{2}(\Omega)}^{2} .
\end{aligned}
$$

Via integration of the last inequality, it follows that

$$
\begin{aligned}
\| T_{h}(t & =s)\left\|_{L^{2}(\Omega)}^{2}+\lambda\right\| T_{h} \|_{L^{2}\left(0, t, H_{0, \Gamma_{1}}^{1}(\Omega)\right)}^{2} \\
& \leq \frac{(\alpha \rho)^{2}}{\lambda}\left\|C_{h}\right\|_{L^{2}\left(0, t, L^{2}(\Omega)\right)}^{2}+\left\|T_{h}^{0}\right\|_{L^{2}(\Omega)}^{2} .
\end{aligned}
$$

Thus, using inequality (23), we get

$$
\begin{aligned}
\| T_{h}(t & =s)\left\|_{L^{2}(\Omega)}^{2}+\lambda\right\| T_{h} \|_{L^{2}\left(0, t, H_{0, \Gamma_{1}}^{1}(\Omega)\right)}^{2} \\
& \leq \frac{(\alpha \rho)^{2}}{2 \lambda \eta}\left\|C_{h}^{0}\right\|_{L^{2}(\Omega)}^{2}+\left\|T_{h}^{0}\right\|_{L^{2}(\Omega)}^{2} .
\end{aligned}
$$

It leads to

$$
\left\|T_{h}\right\|_{L^{\infty}\left(0, t, L^{2}(\Omega)\right)}^{2} \leq \frac{(\alpha \rho)^{2}}{2 \lambda \eta}\left\|C_{h}^{0}\right\|_{L^{2}(\Omega)}^{2}+\left\|T_{h}^{0}\right\|_{L^{2}(\Omega)}^{2} .
$$

From (28) and (29), it follows that

$$
\begin{gathered}
\left\|T_{h}\right\|_{L^{\infty}\left(0, t, L^{2}(\Omega)\right)}^{2}+\lambda\left\|T_{h}\right\|_{L^{2}\left(0, t, H_{0, \Gamma_{1}}^{1}(\Omega)\right)}^{2} \\
\quad \leq \frac{(\alpha \rho)^{2}}{\lambda \eta}\left\|C_{h}^{0}\right\|_{L^{2}(\Omega)}^{2}+2\left\|T_{h}^{0}\right\|_{L^{2}(\Omega)}^{2} .
\end{gathered}
$$

For the vorticity, we have the following.

Lemma 4. For any local solution $\omega_{h}$ of the problem $\left(\mathscr{P}_{h}\right)$, one has the estimate

$$
\begin{aligned}
& \left\|\omega_{h}\right\|_{L^{\infty}\left(0, t, L^{2}(\Omega)\right)}^{2}+\mu_{p}\left\|\omega_{h}\right\|_{L^{2}\left(0, t, L^{2}(\Omega)\right)}^{2} \\
& \quad \leq \frac{2 \alpha \rho^{2}}{\lambda^{2} \eta \mu_{p}}\left\|C_{h}^{0}\right\|_{L^{2}(\Omega)}^{2}+\frac{4}{\lambda \mu_{p}}\left\|T_{h}^{0}\right\|_{L^{2}(\Omega)}^{2}+2\left\|\omega_{h}^{0}\right\|_{L^{2}(\Omega)}^{2} .
\end{aligned}
$$

Proof. By choosing $v_{h}=\omega_{h}$, as test function in the first equation of the problem $\left(\mathscr{P}_{h}\right)$, we have

$$
\frac{1}{2} \frac{d}{d t}\left\|\omega_{h}\right\|_{L^{2}(\Omega)}^{2}+\mu_{p}\left\|\omega_{h}\right\|_{L^{2}(\Omega)}^{2} \leq\left\|\nabla T_{h}\right\|_{L^{2}(\Omega)}\left\|\omega_{h}\right\|_{L^{2}(\Omega)} .
$$

Then, by using triangular inequality, we get

$$
\frac{1}{2} \frac{d}{d t}\left\|\omega_{h}\right\|_{L^{2}(\Omega)}^{2}+\frac{\mu_{p}}{2}\left\|\omega_{h}\right\|_{L^{2}(\Omega)}^{2} \leq \frac{1}{2 \mu_{p}}\left\|\nabla T_{h}\right\|_{L^{2}(\Omega)}^{2} .
$$

While integrating the last inequality, we obtain

$$
\begin{aligned}
\| \omega_{h}(t & =s)\left\|_{L^{2}(\Omega)}^{2}+\mu_{p}\right\| \omega_{h} \|_{L^{2}\left(0, t, L^{2}(\Omega)\right)}^{2} \\
& \leq \frac{1}{\mu_{p}}\left\|T_{h}\right\|_{L^{2}\left(0, t, H_{0, \Gamma_{1}}^{1}(\Omega)\right)}^{2}+\left\|\omega_{h}^{0}\right\|_{L^{2}(\Omega)}^{2} .
\end{aligned}
$$

However, using Lemma 3, we have

$$
\begin{aligned}
& \left\|\omega_{h}(t=s)\right\|_{L^{2}(\Omega)}^{2}+\mu_{p}\left\|\omega_{h}\right\|_{L^{2}\left(0, t, L^{2}(\Omega)\right)}^{2} \\
& \quad \leq \frac{\alpha \rho^{2}}{\lambda^{2} \eta \mu_{p}}\left\|C_{h}^{0}\right\|_{L^{2}(\Omega)}^{2}+\frac{2}{\lambda \mu_{p}}\left\|T_{h}^{0}\right\|_{L^{2}(\Omega)}^{2}+\left\|\omega_{h}^{0}\right\|_{L^{2}(\Omega)}^{2} .
\end{aligned}
$$

It leads to

$$
\begin{aligned}
\left\|\omega_{h}\right\|_{L^{\infty}\left(0, t, L^{2}(\Omega)\right)}^{2} \leq & \frac{\alpha \rho^{2}}{\lambda^{2} \eta \mu_{p}}\left\|C_{h}^{0}\right\|_{L^{2}(\Omega)}^{2} \\
& +\frac{2}{\lambda \mu_{p}}\left\|T_{h}^{0}\right\|_{L^{2}(\Omega)}^{2}+\left\|\omega_{h}^{0}\right\|_{L^{2}(\Omega)}^{2} .
\end{aligned}
$$

From (35) and (36), we obtain

$$
\begin{aligned}
& \left\|\omega_{h}\right\|_{L^{\infty}\left(0, t, L^{2}(\Omega)\right)}^{2}+\mu_{p}\left\|\omega_{h}\right\|_{L^{2}\left(0, t, L^{2}(\Omega)\right)}^{2} \\
& \quad \leq \frac{2 \alpha \rho^{2}}{\lambda^{2} \eta \mu_{p}}\left\|C_{h}^{0}\right\|_{L^{2}(\Omega)}^{2}+\frac{4}{\lambda \mu_{p}}\left\|T_{h}^{0}\right\|_{L^{2}(\Omega)}^{2}+2\left\|\omega_{h}^{0}\right\|_{L^{2}(\Omega)}^{2} .
\end{aligned}
$$


Finally, for the stream function, we have the following.

Lemma 5. For any local solution $\psi_{h}$ of the problem $\left(\mathscr{P}_{h}\right)$, one has the estimate

$$
\begin{aligned}
\left\|\psi_{h}\right\|_{L^{2}\left(0, t, W^{1,4}(\Omega)\right)}^{2} \lesssim & \frac{2 \alpha \rho^{2}}{\lambda^{2} \eta \mu_{p}^{2}}\left\|C_{h}^{0}\right\|_{L^{2}(\Omega)}^{2} \\
& +\frac{4}{\lambda \mu_{p}^{2}}\left\|T_{h}^{0}\right\|_{L^{2}(\Omega)}^{2}+\frac{2}{\mu_{p}}\left\|\omega_{h}^{0}\right\|_{L^{2}(\Omega)}^{2} .
\end{aligned}
$$

Proof. Let $\psi_{h}$ be the solution of the problem $\left(\mathscr{P}_{h}\right)$. From [8, 9], we have

$$
\left|\psi_{h}\right|_{W^{1,4}(\Omega)} \lesssim \sup _{\substack{v \in \stackrel{\circ}{W}^{1,4 / 3}(\Omega)\\}} \frac{\left\langle\nabla\left(\psi_{h}\right), \nabla v\right\rangle}{|v|_{W^{1,4 / 3}(\Omega)}} .
$$

However,

$$
\left\langle\nabla\left(\psi_{h}\right), \nabla v\right\rangle=\left\langle\nabla\left(\psi_{h}\right), \nabla\left(\Pi_{h} v\right)\right\rangle,
$$

where $\Pi_{h}$ is the projection operator defined from $W^{1,4 / 3}(\Omega)$ onto $X_{h}$, such as

$$
\forall v \in W^{1,4 / 3}(\Omega), \quad\left\langle\nabla w_{h}, \nabla\left(\Pi_{h} v\right)-\nabla v\right\rangle=0, \quad \forall w_{h} \in X_{h} .
$$

By choosing $v_{h}=\psi_{h}$, as test function in the last equation of the problem $\left(\mathscr{P}_{h}\right)$, we get

$$
\left\langle\nabla \psi_{h}, \nabla \Pi_{h} v\right\rangle=\left\langle\omega_{h}, \Pi_{h} v\right\rangle .
$$

Therefore,

$$
\left\langle\nabla \psi_{h}, \nabla v\right\rangle \leq\left\|\omega_{h}\right\|_{L^{2}(\Omega)}\left\|\Pi_{h} v\right\|_{L^{2}(\Omega)} .
$$

Due to the embedding of $W^{1,4 / 3}(\Omega)$ onto $L^{2}(\Omega)$, we have

$$
\left\|\Pi_{h} v\right\|_{L^{2}(\Omega)} \lesssim\left|\Pi_{h} v\right|_{W^{1,4 / 3}(\Omega)},
$$

and also, due to the propriety of the operator $\Pi_{h}$, we have

$$
\left\|\Pi_{h} v\right\|_{W^{1,4 / 3}(\Omega)} \lesssim|v|_{W^{1,4 / 3}(\Omega)},
$$

so

$$
\sup _{\substack{1,4 / 3 \\ v \in W^{1,4 / 3)}}} \frac{\left\langle\nabla \psi_{h}, \nabla v\right\rangle}{|v|_{W^{1,4 / 3}(\Omega)}} \lesssim\left\|\omega_{h}\right\|_{L^{2}(\Omega)} .
$$

We conclude that

$$
\left|\psi_{h}\right|_{W^{1,4}(\Omega)} \lesssim\left\|\omega_{h}\right\|_{L^{2}(\Omega)} .
$$

Then, we get

$$
\left\|\psi_{h}\right\|_{L^{2}\left(0, t,\left(W^{1,4}(\Omega)\right)\right)} \lesssim\left\|\omega_{h}\right\|_{L^{2}\left(0, t, L^{2}(\Omega)\right)} .
$$

Using Lemma 4, we conclude that

$$
\begin{aligned}
& \left\|\psi_{h}\right\|_{L^{2}\left(0, t, \stackrel{1}{W}^{1,4}(\Omega)\right)}^{2} \\
& \quad \leq\left(\frac{2 \alpha \rho^{2}}{\lambda^{2} \eta \mu_{p}^{2}}\left\|C_{h}^{0}\right\|_{L^{2}(\Omega)}^{2}+\frac{4}{\lambda \mu_{p}^{2}}\left\|T_{h}^{0}\right\|_{L^{2}(\Omega)}^{2}+\frac{2}{\mu_{p}}\left\|\omega_{h}^{0}\right\|_{L^{2}(\Omega)}^{2}\right) .
\end{aligned}
$$

Now, we are able to prove the main theorem of this section more precisely.

Theorem 6. The problem $\left(\mathscr{P}_{h}\right)$ admits at least a solution

$$
\begin{aligned}
& \left(\omega_{h}, \psi_{h}, C_{h}, T_{h}\right) \\
& \quad \in H^{1}\left(0, t, M_{h}\right) \times L^{2}\left(0, t, X_{h}\right) \times\left(\mathscr{C}^{1}\left(0, t, W_{h}\right)\right)^{2} .
\end{aligned}
$$

Proof. Indeed, it is obvious that the problem $\left(\mathscr{P}_{h}\right)$ admits a local solution in the interval $\left(0, t_{h}\right)$. For $t_{h}$ which is rather small, Lemmas 2, 3, 4, and 5 show that this solution can be defined on the interval $(0, t)$ for $t>0$.

\section{A Priori Error Estimations}

In this section, we prove some error estimates on the stream function, on the vorticity, on the temperature, and on the concentration. Subsequently, we assume that there exists $0<$ $\sigma \leq 1$, such as

$$
\begin{gathered}
\omega \in L^{2}\left(0, t, H^{\sigma}(\Omega)\right) \cap H^{1}\left(0, t, H^{\sigma}(\Omega)\right), \\
T, C \in L^{2}\left(0, t, H^{1+\sigma}(\Omega)\right) \cap H^{1}\left(0, t, H^{\sigma}(\Omega)\right), \\
\psi \in \stackrel{\circ}{W}^{1,4}(\Omega) \cap W^{1+\sigma, 4}(\Omega) .
\end{gathered}
$$

Lemma 7. For any $\omega$ and $\omega_{h}$ solutions of the problems $(\mathscr{P})$ and $\left(\mathscr{P}_{h}\right)$, respectively, one has

$$
\begin{aligned}
\| \omega- & \omega_{h} \|_{L^{2}\left(0, t, L^{2}(\Omega)\right)} \\
\lesssim & h^{\sigma}\left(\|\omega\|_{L^{2}\left(0, t, H^{\sigma}(\Omega)\right)}+\|\omega\|_{H^{1}\left(0, t, H^{\sigma}(\Omega)\right)}+\|T\|_{L^{2}\left(0, t, H^{1+\sigma}(\Omega)\right)}\right) \\
& +\frac{\sqrt{3} \beta g}{\mu_{p}}\left\|T-T_{h}\right\|_{L^{2}\left(0, t, H_{0, \Gamma_{1}}^{1}(\Omega)\right)} .
\end{aligned}
$$

Proof. For $\omega$ and $\omega_{h}$, respectively, solutions of the continuous and the semidiscrete problem, we have

$$
\begin{aligned}
\left(\partial_{t} \omega\right. & \left.-\partial_{t} \omega_{h}, v_{h}\right)_{\mathcal{\kappa}}+\mu_{p}\left(\omega-\omega_{h}, v_{h}\right)_{\kappa} \\
& =\left(f(T)-f\left(T_{h}\right), v_{h}\right)_{\mathcal{K}}, \quad \forall v_{h} \in X_{h},
\end{aligned}
$$

where $\kappa$ is an element of the uniform meshes family $\tau_{h}$. Let $\bar{\omega}_{h}$ be the approximation of $\omega$ in $X_{h}$ defined by

$$
\left.\bar{\omega}_{h}\right|_{\kappa}=\frac{1}{\operatorname{mes}(\kappa)} \int_{\kappa} \omega d x .
$$

Hence, for all $0<\sigma<1$ and $\omega \in H^{\sigma}(\Omega)$, we have the following result:

$$
\left\|\omega-\bar{\omega}_{h}\right\|_{L^{2}(\kappa)} \lesssim h_{\kappa}^{\sigma}\|\omega\|_{H^{\sigma}(\kappa)},
$$

where $h_{\kappa}$ is the diameter of the element $\kappa$.

By using equality (53) and setting $v_{h}=\bar{\omega}_{h}-\omega_{h}$, we obtain

$$
\begin{aligned}
\left(\partial_{t} \bar{\omega}_{h}-\partial_{t} \omega_{h}, \bar{\omega}_{h}-\omega_{h}\right)_{\kappa}+\mu_{p}\left(\bar{\omega}_{h}-\omega_{h}, \bar{\omega}_{h}-\omega_{h}\right)_{\kappa} \\
=-\left(\partial_{t} \omega-\partial_{t} \bar{\omega}_{h}, \bar{\omega}_{h}-\omega_{h}\right)_{\kappa}+\mu_{p}\left(\bar{\omega}_{h}-\omega, \bar{\omega}_{h}-\omega_{h}\right)_{\kappa} \\
+\left(f(T)-f\left(T_{h}\right), \bar{\omega}_{h}-\omega_{h}\right)_{\kappa} .
\end{aligned}
$$


Therefore, we have

$$
\begin{aligned}
& \frac{1}{2} \frac{d}{d t}\left\|\bar{\omega}_{h}-\omega_{h}\right\|_{L^{2}(\kappa)}^{2}+\mu_{p}\left\|\bar{\omega}_{h}-\omega_{h}\right\|_{L^{2}(\kappa)}^{2} \\
& \leq\left(\left\|\partial_{t} \omega-\partial_{t} \bar{\omega}_{h}\right\|_{L^{2}(\kappa)}+\beta g\left\|\nabla\left(T-T_{h}\right)\right\|_{L^{2}(\kappa)}\right) \\
& \times\left\|\bar{\omega}_{h}-\omega_{h}\right\|_{L^{2}(\kappa)} \\
&+\mu_{p}\left\|\omega-\bar{\omega}_{h}\right\|_{L^{2}(\kappa)}\left\|\bar{\omega}_{h}-\omega_{h}\right\|_{L^{2}(\kappa)} .
\end{aligned}
$$

Then,

$$
\begin{aligned}
& \frac{1}{2} \frac{d}{d t}\left\|\bar{\omega}_{h}-\omega_{h}\right\|_{L^{2}(\kappa)}^{2}+\mu_{p}\left\|\bar{\omega}_{h}-\omega_{h}\right\|_{L^{2}(\kappa)}^{2} \\
& \leq\left(\left\|\partial_{t} \omega-\bar{\partial}_{t} \omega_{h}\right\|_{L^{2}(\kappa)}+\beta g\left\|\nabla\left(T-T_{h}\right)\right\|_{L^{2}(\kappa)}\right) \\
& \times\left\|\bar{\omega}_{h}-\omega_{h}\right\|_{L^{2}(\kappa)} \\
&+\mu_{p}\left\|\omega-\bar{\omega}_{h}\right\|_{L^{2}(\kappa)}\left\|\bar{\omega}_{h}-\omega_{h}\right\|_{L^{2}(\kappa)} .
\end{aligned}
$$

However, by using the triangular inequality, we can write

$$
\begin{gathered}
\frac{1}{2} \frac{d}{d t}\left\|\bar{\omega}_{h}-\omega_{h}\right\|_{L^{2}(\kappa)}^{2}+\mu_{p}\left\|\bar{\omega}_{h}-\omega_{h}\right\|_{L^{2}(\kappa)}^{2} \\
\leq \frac{3}{2 \mu_{p}}\left\|\partial_{t} \omega-\bar{\partial}_{t} \omega_{h}\right\|_{L^{2}(\kappa)}^{2}+\frac{3(\beta g)^{2}}{2 \mu_{p}}\left\|\nabla\left(T-T_{h}\right)\right\|_{L^{2}(\kappa)}^{2} \\
\quad+\frac{3 \mu_{p}}{2}\left\|\bar{\omega}_{h}-\omega\right\|_{L^{2}(\kappa)}^{2}+\frac{\mu_{p}}{2}\left\|\bar{\omega}_{h}-\omega\right\|_{L^{2}(\kappa)}^{2} .
\end{gathered}
$$

It follows that

$$
\begin{aligned}
\frac{1}{2} \frac{d}{d t}\left\|\bar{\omega}_{h}-\omega_{h}\right\|_{L^{2}(\kappa)}^{2}+\frac{\mu_{p}}{2}\left\|\bar{\omega}_{h}-\omega_{h}\right\|_{L^{2}(\kappa)}^{2} \\
\leq \frac{3}{2 \mu_{p}}\left\|\partial_{t} \omega-\bar{\partial}_{t} \omega_{h}\right\|_{L^{2}(\kappa)}^{2} \\
\quad+\frac{3 \beta^{2} g^{2}}{2 \mu_{p}}\left\|T-T_{h}\right\|_{H_{0, \Gamma_{1}}^{1}(\kappa)}^{2}+\frac{3 \mu_{p}}{2}\left\|\bar{\omega}_{h}-\omega\right\|_{L^{2}(\kappa)}^{2} .
\end{aligned}
$$

However, for all $0<\sigma<1$ and $\omega \in H^{1}\left(0, t, H^{\sigma}(\kappa)\right)$, we have

$$
\left\|\partial_{t} \omega-\bar{\partial}_{t} \omega_{h}\right\|_{L^{2}(\kappa)}^{2} \lesssim h_{\kappa}^{2 \sigma}\left\|\partial_{t} \omega\right\|_{H^{\sigma}(\kappa)}^{2} .
$$

While multiplying (60) by $2 / \mu$ and using (55) and (61), we obtain

$$
\begin{aligned}
\left\|\bar{\omega}_{h}-\omega_{h}\right\|_{L^{2}(\kappa)}^{2} \lesssim & h_{\kappa}^{2 \sigma}\left(\|\omega\|_{H^{\sigma}(\kappa)}+\left\|\partial_{t} \omega\right\|_{H^{\sigma}(\kappa)}\right)^{2} \\
& +\frac{3(\beta g)^{2}}{\mu_{p}^{2}}\left\|T-T_{h}\right\|_{H_{0, \Gamma_{1}}^{1}(\kappa)} .
\end{aligned}
$$

Finally, by integrating and using Jensen's inequality, we get

$$
\begin{aligned}
\| \bar{\omega}_{h}- & \omega_{h} \|_{L^{2}\left(0, t, L^{2}(\Omega)\right)} \\
\lesssim & h^{\sigma}\left(\|\omega\|_{L^{2}\left(0, t, H^{\sigma}(\Omega)\right)}+\|\omega\|_{H^{1}\left(0, t, H^{\sigma}(\Omega)\right)}\right) \\
& +\frac{\sqrt{3} \beta g}{\mu_{p}}\left\|T-T_{h}\right\|_{L^{2}\left(0, t, H_{0, \Gamma_{1}}^{1}(\Omega)\right)} .
\end{aligned}
$$

However,

$$
\begin{aligned}
\left\|\omega-\omega_{h}\right\|_{L^{2}\left(0, t, L^{2}(\Omega)\right)} \leq & \left\|\bar{\omega}_{h}-\omega_{h}\right\|_{L^{2}\left(0, t, L^{2}(\Omega)\right)} \\
& +\left\|\omega-\bar{\omega}_{h}\right\|_{L^{2}\left(0, t, L^{2}(\Omega)\right)} .
\end{aligned}
$$

We conclude that

$$
\begin{aligned}
\left\|\omega-\omega_{h}\right\|_{L^{2}\left(0, t, L^{2}(\Omega)\right)} \lesssim & h^{\sigma}\left(\|\omega\|_{L^{2}\left(0, t, H^{\sigma}(\Omega)\right)}+\|\omega\|_{H^{1}\left(0, t, H^{\sigma}(\Omega)\right)}\right) \\
& +\frac{\sqrt{3} \beta g}{\mu_{p}}\left\|T-T_{h}\right\|_{L^{2}\left(0, t, H_{0, \Gamma_{1}}^{1}(\Omega)\right)} .
\end{aligned}
$$

Lemma 8. For any $\psi$ and $\psi_{h}$ solution of the problems $(\mathscr{P})$ and $\left(\mathscr{P}_{h}\right)$, respectively, one has

$$
\begin{aligned}
\left\|\psi-\psi_{h}\right\|_{L^{2}\left(0, t, \stackrel{\circ}{W}^{1,4}(\Omega)\right)} \leq & h^{\sigma}\|\psi\|_{L^{2}\left(0, t,\left(W^{1+\sigma, 4}(\Omega)\right)\right)} \\
& +\left\|\omega-\omega_{h}\right\|_{L^{2}\left(0, t, L^{2}(\Omega)\right)} .
\end{aligned}
$$

Proof. Let $\psi$ and $\psi_{h}$ be the solution of the problems $\left(\mathscr{P}_{v}\right)$ and $\left(\mathscr{P}_{h}\right)$, respectively. From $[8,9]$, we have

$$
\left|\psi-\psi_{h}\right|_{W^{1,4}(\Omega)} \lesssim \sup _{\substack{i, W^{1,4 / 3} \\(\Omega)}} \frac{\left\langle\nabla\left(\psi-\psi_{h}\right), \nabla v\right\rangle}{|v|_{W^{1,4 / 3}(\Omega)}} .
$$

However,

$$
\begin{aligned}
\left\langle\nabla\left(\psi-\psi_{h}\right), \nabla v\right\rangle= & \left\langle\nabla\left(\psi-\psi_{h}\right), \nabla\left(v-\Pi_{h} v\right)\right\rangle \\
& +\left\langle\nabla\left(\psi-\psi_{h}\right), \nabla\left(\Pi_{h} v\right)\right\rangle,
\end{aligned}
$$

where $\Pi_{h}$ is the projection operator verifying (41). Moreover, we have

$$
\begin{gathered}
\left\langle\nabla\left(R_{h} \psi-\psi_{h}\right), \nabla\left(\Pi_{h} v-v\right)\right\rangle=0, \\
\left\langle\nabla\left(R_{h} \psi-\psi\right), \nabla\left(\Pi_{h} v\right)\right\rangle=0 .
\end{gathered}
$$

Then, we get

$$
\begin{aligned}
\left\langle\nabla\left(\psi-\psi_{h}\right), \nabla v\right\rangle= & \left\langle\nabla\left(\psi-R_{h} \psi\right), \nabla\left(v-\Pi_{h} v\right)\right\rangle \\
& +\left\langle\nabla\left(\psi-\psi_{h}\right), \nabla\left(\Pi_{h} v\right)\right\rangle .
\end{aligned}
$$

By choosing $v_{h}=\Pi_{h} v$ as test function in the last equation of the problem $\left(P_{h}\right)$, we obtain

$$
\left\langle\nabla\left(\psi-\psi_{h}\right), \nabla v\right\rangle=\left\langle\nabla\left(\psi-R_{h} \psi\right), \nabla v\right\rangle+\left\langle\omega-\omega_{h}, \Pi_{h} v\right\rangle .
$$

It leads to

$$
\begin{gathered}
\left\langle\nabla\left(\psi-R_{h} \psi\right), \nabla v\right\rangle \leq\left|\psi-R_{h} \psi\right|_{W^{1,4}(\Omega)}|v|_{W^{1,4 / 3}(\Omega)}, \\
\left\langle\omega-\omega_{h}, \Pi_{h} v\right\rangle \leq\left\|\omega-\omega_{h}\right\|_{L^{2}(\Omega)}\left\|\Pi_{h} v\right\|_{L^{2}(\Omega)} .
\end{gathered}
$$

On the other hand, due to the embedding of $W^{1,4 / 3}(\Omega)$ onto $L^{2}(\Omega)$, we have

$$
\left\|\Pi_{h} v\right\|_{L^{2}(\Omega)} \lesssim\left|\Pi_{h} v\right|_{W^{1,4 / 3}(\Omega)} .
$$


In addition,

$$
\left|\Pi_{h} v\right|_{W^{1,4 / 3}(\Omega)} \lesssim|v|_{W^{1,4 / 3}(\Omega)},
$$

so

$$
\sup _{v \in W^{1,4 / 3}(\Omega)} \frac{\left\langle\omega-\omega_{h}, \Pi_{h} v\right\rangle}{|v|_{W^{1,4 / 3}(\Omega)}} \leqslant\left\|\omega-\omega_{h}\right\|_{L^{2}(\Omega)} .
$$

From inequalities (67) and (72), we have

$$
\left|\psi-\psi_{h}\right|_{W^{1,4}(\Omega)} \lesssim\left|\psi-R_{h} \psi\right|_{W^{1,4}(\Omega)}+\left\|\omega-\omega_{h}\right\|_{L^{2}(\Omega)} .
$$

We conclude that

$$
\begin{aligned}
& \left\|\psi-\psi_{h}\right\|_{L^{2}\left(0, t, \stackrel{1}{W}^{1,4}(\Omega)\right)} \\
& \quad \lesssim h^{\sigma}\|\psi\|_{L^{2}\left(0, t,\left(W^{1+\sigma, 4}(\Omega)\right)\right)}+\left\|\omega-\omega_{h}\right\|_{L^{2}\left(0, t, L^{2}(\Omega)\right)} .
\end{aligned}
$$
ture.

Also, we have the following error estimate for tempera-

Lemma 9. If we assume that the hypothesis $\left(H_{1}\right)$ is verified, then one has

$$
\begin{aligned}
\left\|T-T_{h}\right\|_{L^{2}\left(0, t, H_{0, \Gamma_{1}}^{1}(\Omega)\right)} & \\
\lesssim & h^{\sigma}\left(\|T\|_{H^{1}\left(0, t, H^{\sigma}(\Omega)\right)}+\|T\|_{L^{2}\left(0, t, H^{1+\sigma}(\Omega)\right)}\right) \\
& +\frac{\sqrt{5} \alpha \rho^{2}}{\delta_{1} \lambda}\left\|C-C_{h}\right\|_{L^{2}\left(0, t, H_{0, \Gamma_{1}}^{1}(\Omega)\right)} \\
& +\frac{\sqrt{5} N N_{T}}{\delta_{1} \lambda}\left\|\psi-\psi_{h}\right\|_{L^{2}(0, t, \stackrel{\circ}{W} \stackrel{1,4}{(\Omega)})} .
\end{aligned}
$$

Proof. For $T$ solution of the problem $(\mathscr{P})$ and $T_{h}$ solution of the problem $\left(\mathscr{P}_{h}\right)$, we have

$$
\begin{aligned}
\left(\partial_{t} T-\right. & \left.\partial_{t} T_{h}, \phi_{h}\right)+\lambda a\left(T-T_{h}, \phi_{h}\right) \\
& +a_{1}\left(\psi-\psi_{h}, T, \phi_{h}\right)+a_{1}\left(\psi_{h}, T-T_{h}, \phi_{h}\right) \\
= & k\left(C, T, \phi_{h}\right)-k\left(C_{h}, T_{h}, \phi_{h}\right) .
\end{aligned}
$$

Therefore, using the propriety of the operator $i_{h}$, we have

$$
a\left(i_{h} T-T, \phi_{h}\right)=0 \text {. }
$$

So,

$$
\begin{aligned}
\left(i_{h} \partial_{t} T\right. & \left.-\partial_{t} T_{h}, \phi_{h}\right)+\lambda a\left(i_{h} T-T_{h}, \phi_{h}\right) \\
= & \left(i_{h} \partial_{t} T-\partial_{t} T, \phi_{h}\right)-a_{1}\left(\psi-\psi_{h}, T, \phi_{h}\right) \\
& \quad-a_{1}\left(\psi_{h}, T-T_{h}, \phi_{h}\right)+k\left(C, T, \phi_{h}\right)-k\left(C_{h}, T_{h}, \phi_{h}\right) .
\end{aligned}
$$

By setting $\phi_{h}=i_{h} T-T_{h}$, we obtain

$$
\begin{aligned}
\frac{1}{2} \frac{d}{d t}\left\|i_{h} T-T_{h}\right\|_{L^{2}(\Omega)}^{2}+\lambda\left\|\nabla\left(i_{h} T-T_{h}\right)\right\|_{L^{2}(\Omega)}^{2} \\
=-\left(\partial_{t} T-i_{h} \partial_{t} T, \phi_{h}\right)-a_{1}\left(\psi-\psi_{h}, T, \phi_{h}\right) \\
-a_{1}\left(\psi_{h}, T-i_{h} T, i_{h} T-T_{h}\right)+k\left(C, T, \phi_{h}\right) \\
-k\left(C_{h}, T_{h}, \phi_{h}\right)
\end{aligned}
$$

Then, we get

$$
\begin{aligned}
& \frac{1}{2} \frac{d}{d t}\left\|i_{h} T-T_{h}\right\|_{L^{2}(\Omega)}^{2}+\lambda\left\|\nabla\left(i_{h} T-T_{h}\right)\right\|_{L^{2}(\Omega)}^{2} \\
& \leq\left(\rho\left\|\partial_{t} T-i_{h} \partial_{t} T\right\|_{L^{2}(\Omega)}+N N_{T}\left\|\psi-\psi_{h}\right\|_{\dot{W}^{1,4}(\Omega)}\right. \\
& \left.\quad+N M_{1}\left\|\nabla\left(T-i_{h} T\right)\right\|_{L^{2}(\Omega)}\right)\left\|\nabla\left(i_{h} T-T_{h}\right)\right\|_{L^{2}(\Omega)} \\
& +\left|k\left(C, T, \phi_{h}\right)+k\left(C_{h}, T_{h}, \phi_{h}\right)\right| .
\end{aligned}
$$

We have also

$$
\begin{aligned}
&\left|k\left(C, T, \phi_{h}\right)-k\left(C_{h}, T_{h}, \phi_{h}\right)\right| \\
& \leq \alpha \rho^{2}\left\|C-C_{h}\right\|_{H_{0, \Gamma_{1}}^{1}(\Omega)}\left\|\phi_{h}\right\|_{H_{0, \Gamma_{1}}^{1}(\Omega)} \\
& \quad+N_{C} \alpha \frac{E}{R T_{i}^{2}} \rho^{2}\left\|T-T_{h}\right\|_{H_{0, \Gamma_{1}}^{1}(\Omega)}\left\|\phi_{h}\right\|_{H_{0, \Gamma_{1}}^{1}(\Omega)} .
\end{aligned}
$$

From (83) and (84), we get

$$
\begin{aligned}
& \frac{1}{2} \frac{d}{d t}\left\|i_{h} T-T_{h}\right\|_{L^{2}(\Omega)}^{2}+\lambda\left\|\nabla\left(i_{h} T-T_{h}\right)\right\|_{L^{2}(\Omega)}^{2} \\
& \leq\left(\rho\left\|\partial_{t} T-i_{h} \partial_{t} T\right\|_{L^{2}(\Omega)}+N N_{T}\left\|\psi-\psi_{h}\right\|_{W^{1,4}(\Omega)}\right. \\
& \left.\quad+N M_{1}\left\|\nabla\left(T-i_{h} T\right)\right\|_{L^{2}(\Omega)}\right)\left\|\nabla\left(i_{h} T-T_{h}\right)\right\|_{L^{2}(\Omega)} \\
& +\left(\alpha \rho^{2}\left\|C-C_{h}\right\|_{H_{0, \Gamma_{1}}^{1}(\Omega)}+N_{C} \alpha \frac{E}{R T_{i}^{2}} \rho^{2}\left\|T-T_{h}\right\|_{H_{0, \Gamma_{1}}^{1}(\Omega)}\right) \\
& \quad \times\left\|\nabla\left(i_{h} T-T_{h}\right)\right\|_{L^{2}(\Omega)} .
\end{aligned}
$$

Then, we deduce

$$
\begin{aligned}
\frac{1}{2} \frac{d}{d t}\left\|i_{h} T-T_{h}\right\|_{L^{2}(\Omega)}^{2}+\frac{\lambda}{2}\left\|\nabla\left(i_{h} T-T_{h}\right)\right\|_{L^{2}(\Omega)}^{2} \\
\leq \frac{5 \rho^{2}}{2 \lambda}\left\|\partial_{t} T-i_{h} \partial_{t} T\right\|_{L^{2}(\Omega)}^{2} \\
+\frac{5 N^{2} M_{1}^{2}}{2 \lambda}\left\|\nabla\left(T-i_{h} T\right)\right\|_{L^{2}(\Omega)}^{2} \\
+\frac{5 \alpha^{2} \rho^{4}}{2 \lambda}\left\|\nabla\left(C-C_{h}\right)\right\|_{L^{2}(\Omega)}^{2} \\
+\frac{5 N^{2} N_{T}^{2}}{2 \lambda}\left\|\psi-\psi_{h}\right\|_{\dot{W}^{1,4}}^{2}(\Omega) \\
+\frac{5 N_{C}^{2} \alpha^{2} E^{2} \rho^{4}}{2 \lambda R^{2} T_{i}^{4}}\left\|\nabla\left(T-T_{h}\right)\right\|_{L^{2}(\Omega)}^{2} .
\end{aligned}
$$


By multiplying the last inequality by $2 / \lambda$, we obtain

$$
\begin{aligned}
& \left\|i_{h} T-T_{h}\right\|_{H_{0, \Gamma_{1}}^{1}(\Omega)}^{2} \leq \frac{5 \rho^{2}}{\lambda^{2}}\left\|\partial_{t} T-i_{h} \partial_{t} T\right\|_{L^{2}(\Omega)}^{2} \\
& +\frac{5 N^{2} M_{1}^{2}}{\lambda^{2}}\left\|\nabla\left(T-i_{h} T\right)\right\|_{L^{2}(\Omega)}^{2} \\
& +\frac{5 \alpha^{2} \rho^{4}}{\lambda^{2}}\left\|\nabla\left(C-C_{h}\right)\right\|_{L^{2}(\Omega)}^{2} \\
& +\frac{5 N^{2} N_{T}^{2}}{\lambda^{2}}\left\|\psi-\psi_{h}\right\|_{\stackrel{\circ}{\circ}^{1,4}(\Omega)}^{2} \\
& +5 \frac{N_{C}^{2}(\alpha E)^{2} \rho^{4}}{R^{2} T_{i}^{4} \lambda^{2}}\left\|\nabla\left(T-T_{h}\right)\right\|_{L^{2}(\Omega)}^{2} .
\end{aligned}
$$

Via integration of the last inequality, we obtain

$$
\begin{aligned}
\| i_{h} T- & T_{h} \|_{L^{2}\left(0, t, H_{0, \Gamma_{1}}^{1}(\Omega)\right)}^{2} \\
\lesssim & \left(h^{\sigma}\|T\|_{H^{1}\left(0, t, H^{\sigma}(\Omega)\right)}+h^{\sigma}\|T\|_{L^{2}\left(0, t, H^{1+\sigma}(\Omega)\right)}\right)^{2} \\
& \left.+\frac{5 \alpha^{2} \rho^{4}}{\lambda^{2}}\left\|C-C_{h}\right\|_{L^{2}\left(0, t, H_{0, \Gamma_{1}}^{1}\right.}^{2}(\Omega)\right) \\
& \left.+\frac{5 N^{2} N_{T}^{2}}{\lambda^{2}}\left\|\psi-\psi_{h}\right\|_{L^{2}\left(0, t, \stackrel{\circ}{W}^{1,4}\right.}^{2}(\Omega)\right) \\
& +5 \frac{N_{C}^{2}(\alpha E)^{2} \rho^{4}}{R^{2} T_{i}^{4} \lambda^{2}}\left\|T-T_{h}\right\|_{L^{2}\left(0, t, H_{0, \Gamma_{1}}^{1}(\Omega)\right)}^{2} .
\end{aligned}
$$

Therefore,

$$
\begin{aligned}
\| i_{h} T- & T_{h} \|_{L^{2}\left(0, t, H_{0, \Gamma_{1}}^{1}(\Omega)\right)} \\
\lesssim & \left(h^{\sigma}\|T\|_{H^{1}\left(0, t, H^{\sigma}(\Omega)\right)}+h^{\sigma}\|T\|_{L^{2}\left(0, t, H^{1+\sigma}(\Omega)\right)}\right) \\
& +\frac{\sqrt{5} \alpha \rho^{2}}{\lambda}\left\|C-C_{h}\right\|_{L^{2}\left(0, t, H_{0, \Gamma_{1}}^{1}(\Omega)\right)} \\
& +\frac{\sqrt{5} N N_{T}}{\lambda}\left\|\psi-\psi_{h}\right\|_{L^{2}\left(0, t, \stackrel{\circ}{W}^{1,4}(\Omega)\right)} \\
& +\frac{\sqrt{5} N_{C} \alpha E \rho^{2}}{R T_{i}^{2} \lambda}\left\|T-T_{h}\right\|_{L^{2}\left(0, t, H_{0, \Gamma_{1}}^{1}(\Omega)\right)} .
\end{aligned}
$$

However,

$$
\left\|T-T_{h}\right\|_{H_{0, \Gamma_{1}}^{1}(\Omega)} \leq\left\|T-i_{h} T\right\|_{H_{0, \Gamma_{1}}^{1}(\Omega)}+\left\|i_{h} T-T_{h}\right\|_{H_{0, \Gamma_{1}}^{1}(\Omega)} .
$$

Then, we have

$$
\left\|T-T_{h}\right\|_{H_{0, \Gamma_{1}}^{1}(\Omega)} \lesssim h^{\sigma}\|T\|_{H^{1+\sigma}(\Omega)}+\left\|i_{h} T-T_{h}\right\|_{H_{0, \Gamma_{1}}^{1}(\Omega)} \cdot
$$

Inequalities (89) and (91) allow us to obtain the following estimate:

$$
\begin{gathered}
\left(1-\frac{\sqrt{5} N_{C} \alpha E \rho^{2}}{R T_{i}^{2} \lambda}\right)\left\|T-T_{h}\right\|_{L^{2}\left(0, t, H_{0, \Gamma_{1}}^{1}(\Omega)\right)} \\
\lesssim h^{\sigma}\left(\|T\|_{H^{1}\left(0, t, H^{\sigma}(\Omega)\right)}+\|T\|_{L^{2}\left(0, t, H^{1+\sigma}(\Omega)\right)}\right) \\
+\frac{\sqrt{5} \alpha \rho^{2}}{\lambda}\left\|C-C_{h}\right\|_{L^{2}\left(0, t, H_{0, \Gamma_{1}}^{1}(\Omega)\right)} \\
+\frac{\sqrt{5} N N_{T}}{\lambda}\left\|\psi-\psi_{h}\right\|_{L^{2}\left(0, t, \stackrel{ }{W}^{1,4}(\Omega)\right)}
\end{gathered}
$$

Finally, if we set

$$
\delta_{1}=1-\frac{\sqrt{5} N_{C} \alpha E \rho^{2}}{R T_{i}^{2} \lambda}>0,
$$

we obtain the following estimate:

$$
\begin{aligned}
\| T- & T_{h} \|_{L^{2}\left(0, t, H_{0, \Gamma_{1}}^{1}(\Omega)\right)} \\
\lesssim & h^{\sigma}\left(\|T\|_{H^{1}\left(0, t, H^{\sigma}(\Omega)\right)}+\|T\|_{L^{2}\left(0, t, H^{1+\sigma}(\Omega)\right)}\right) \\
& +\frac{\sqrt{5} \alpha \rho^{2}}{\delta_{1} \lambda}\left(\left\|C-C_{h}\right\|_{L^{2}\left(0, t, H_{0, \Gamma_{1}}^{1}(\Omega)\right)}\right) \\
& +\frac{\sqrt{5} N N_{T}}{\delta_{1} \lambda}\left\|\psi-\psi_{h}\right\|_{L^{2}(0, t, \stackrel{1}{1,4}(\Omega))} .
\end{aligned}
$$

In addition, we have the following error estimate on concentration.

Lemma 10. For $C$ and $C_{h}$, respectively, solution of the problems $(\mathscr{P})$ and $\left(\mathscr{P}_{h}\right)$, one has

$$
\begin{aligned}
\left\|C-C_{h}\right\|_{L^{2}\left(0, t, H_{0, \Gamma_{1}}^{1}(\Omega)\right)} & \\
\lesssim & h^{\sigma}\left(\|C\|_{H^{1}\left(0, t, H^{\sigma}(\Omega)\right)}+\|C\|_{L^{2}\left(0, t, H^{1+\sigma}(\Omega)\right)}\right) \\
& +\frac{2 \sqrt{2} N N_{C}}{\eta}\left\|\psi-\psi_{h}\right\|_{L^{2}\left(0, t, \stackrel{W}{W}^{1,4}(\Omega)\right)} \\
& +2 \frac{N_{C} \alpha E \rho^{2}}{\eta R T_{i}^{2}}\left\|T-T_{h}\right\|_{L^{2}\left(0, t, H_{0, \Gamma_{1}}^{1}(\Omega)\right)}
\end{aligned}
$$

Proof. By choosing $\chi_{h}=i_{h} C-C_{h}$ as test function, we have

$$
\begin{aligned}
\frac{1}{2} \frac{d}{d t}\left\|i_{h} C-C_{h}\right\|_{0, t, L^{2}(\Omega)}^{2}+\eta\left\|\nabla\left(i_{h} C-C_{h}\right)\right\|_{0, t, L^{2}(\Omega)}^{2} \\
=-\left(\partial_{t} C-i_{h} \partial_{t} C, \chi_{h}\right)-a_{1}\left(\psi-\psi_{h}, C, \chi_{h}\right) \\
-a_{1}\left(\psi_{h}, C-i_{h} C, i_{h} C-C_{h}\right) \\
-k\left(C, T, \chi_{h}\right)+k\left(C_{h}, T_{h}, \chi_{h}\right) .
\end{aligned}
$$


However,

$$
\begin{aligned}
& k\left(C, T, \chi_{h}\right)-k\left(C_{h}, T_{h}, \chi_{h}\right) \\
& =k\left(C, T, \chi_{h}\right)-k\left(i_{h} C, T_{h}, \chi_{h}\right)+k\left(\chi_{h}, T_{h}, \chi_{h}\right), \\
& k\left(C, T, \chi_{h}\right)-k\left(i_{h} C, T_{h}, \chi_{h}\right) \\
& =k\left(C-i_{h} C, T, \chi_{h}\right)-k\left(i_{h} C, T-T_{h}, \chi_{h}\right) \\
& \leq \alpha \rho^{2}\left\|\nabla\left(C-i_{h} C\right)\right\|_{L^{2}(\Omega)}\left\|\nabla \chi_{h}\right\|_{L^{2}(\Omega)} \\
& \quad+\int i_{h} C\left(g(T)-g\left(T_{h}\right)\right) \chi_{h} .
\end{aligned}
$$

Then, we have

$$
\begin{aligned}
k\left(C, T, \chi_{h}\right)-k\left(i_{h} C, T_{h}, \chi_{h}\right) & \\
\leq & \alpha \rho^{2}\left\|C-i_{h} C\right\|_{H_{0, \Gamma_{1}}^{1}(\Omega)}\left\|\chi_{h}\right\|_{H_{0, \Gamma_{1}}^{1}(\Omega)} \\
& \quad+N_{C} \alpha \frac{E}{R T_{i}^{2}} \rho^{2}\left\|T-T_{h}\right\|_{H_{0, \Gamma_{1}}^{1}(\Omega)}\left\|\chi_{h}\right\|_{H_{0, \Gamma_{1}}^{1}(\Omega)} .
\end{aligned}
$$

Using (96) and (98), we obtain

$$
\begin{aligned}
& \frac{1}{2} \frac{d}{d t}\left\|i_{h} C-C_{h}\right\|_{L^{2}(\Omega)}^{2}+\eta\left\|\nabla\left(i_{h} C-C_{h}\right)\right\|_{L^{2}(\Omega)}^{2} \\
& \leq\left(\rho\left\|\partial_{t} C-i_{h} \partial_{t} C\right\|_{L^{2}(\Omega)}+N N_{C}\left\|\psi-\psi_{h}\right\|_{W}^{\circ 1,4}(\Omega)\right. \\
& \left.\quad+N M_{1}\left\|\nabla\left(C-i_{h} C\right)\right\|_{L^{2}(\Omega)}\right)\left\|\nabla\left(i_{h} C-C_{h}\right)\right\|_{L^{2}(\Omega)} \\
& \quad+\left(\alpha \rho^{2}\left\|C-i_{h} C\right\|_{H_{0, \Gamma_{1}}^{1}(\Omega)}+N_{C} \alpha \frac{E}{R T_{i}^{2}} \rho^{2}\left\|\nabla\left(T-T_{h}\right)\right\|_{L^{2}(\Omega)}\right) \\
& \quad \times\left\|\nabla \chi_{h}\right\|_{L^{2}(\Omega)} .
\end{aligned}
$$

By using Young's inequality, we obtain

$$
\begin{aligned}
& \frac{1}{2} \frac{d}{d t}\left\|i_{h} C-C_{h}\right\|_{L^{2}(\Omega)}^{2}+\frac{\eta}{2}\left\|\nabla\left(i_{h} T-T_{h}\right)\right\|_{L^{2}(\Omega)}^{2} \\
& \leq \frac{2 \rho^{2}}{\eta}\left\|\partial_{t} C-i_{h} \partial_{t} C\right\|_{L^{2}(\Omega)}^{2} \\
&+\frac{2 N^{2} M_{1}^{2}}{\eta}\left\|\nabla\left(C-i_{h} C\right)\right\|_{L^{2}(\Omega)}^{2} \\
&+\frac{2 \alpha^{2} \rho^{4}}{\eta}\left\|\nabla\left(C-i_{h} C\right)\right\|_{L^{2}(\Omega)}^{2} \\
&+\frac{2 N^{2} N_{C}^{2}}{\eta}\left\|\psi-\psi_{h}\right\|_{W^{1,4}}^{2}(\Omega) \\
&+N_{C} \alpha \frac{E}{R T_{i}^{2}} \rho^{2}\left\|\nabla\left(T-T_{h}\right)\right\|_{L^{2}(\Omega)}\left\|\nabla \chi_{h}\right\|_{L^{2}(\Omega)} .
\end{aligned}
$$

While multiplying the last inequality by $2 / \eta$ and via integration, we obtain

$$
\begin{aligned}
& \left\|i_{h} C-C_{h}\right\|_{L^{2}\left(0, t, H_{0, \Gamma_{1}}^{1}(\Omega)\right)}^{2} \\
& \leq\left(h^{\sigma}\|C\|_{H^{1}\left(0, t, H^{\sigma}(\Omega)\right)}+h^{\sigma}\|C\|_{L^{2}\left(0, t, H^{1+\sigma}(\Omega)\right)}\right)^{2} \\
& \quad+\frac{4 N^{2} N_{C}^{2}}{\eta^{2}}\left\|\psi-\psi_{h}\right\|_{L^{2}\left(0, t, \stackrel{\circ}{W}^{1,4}(\Omega)\right)}^{2} \\
& +2 \frac{N_{C} \alpha E \rho^{2}}{\eta R T_{i}^{2}}\left\|\nabla\left(T-T_{h}\right)\right\|_{L^{2}\left(0, t, L^{2}(\Omega)\right)}\left\|\nabla \chi_{h}\right\|_{L^{2}\left(0, t, L^{2}(\Omega)\right)}
\end{aligned}
$$

So, we get

$$
\begin{aligned}
\| i_{h} C & -C_{h} \|_{L^{2}\left(0, t, H_{0, \Gamma_{1}}^{1}(\Omega)\right)}^{2} \\
\lesssim & \left(h^{\sigma}\|C\|_{H^{1}\left(0, t, H^{\sigma}(\Omega)\right)}+h^{\sigma}\|C\|_{L^{2}\left(0, t, H^{1+\sigma}(\Omega)\right)}\right)^{2} \\
& +\frac{4 N^{2} N_{C}^{2}}{\eta^{2}}\left\|\psi-\psi_{h}\right\|_{L^{2}\left(0, t, \stackrel{\circ}{W}^{1,4}(\Omega)\right)}^{2} \\
& +2 \frac{N_{C^{2}}^{2} \alpha^{2} E^{2} \rho^{4}}{R^{2} T_{i}^{4} \eta^{2}}\left\|T-T_{h}\right\|_{L^{2}\left(0, t, H_{0, \Gamma_{1}}^{1}(\Omega)\right)}^{2} \\
& +\frac{1}{2}\left\|i_{h} C-C_{h}\right\|_{L^{2}\left(0, t, H_{0, \Gamma_{1}}^{1}(\Omega)\right)}^{2}
\end{aligned}
$$

Finally, by the triangular inequality, we obtain

$$
\begin{aligned}
\| C- & C_{h} \|_{L^{2}\left(0, t, H_{0, \Gamma_{1}}^{1}(\Omega)\right)} \\
\lesssim & h^{\sigma}\left(\|C\|_{H^{1}\left(0, t, H^{\sigma}(\Omega)\right)}+\|C\|_{L^{2}\left(0, t, H^{1+\sigma}(\Omega)\right)}\right) \\
& +\frac{2 \sqrt{2} N N_{C}}{\eta}\left\|\psi-\psi_{h}\right\|_{L^{2}\left(0, t, \stackrel{\circ}{W}^{1,4}(\Omega)\right)} \\
& +2 \frac{N_{C} \alpha E \rho^{2}}{\eta R T_{i}^{2}}\left\|T-T_{h}\right\|_{L^{2}\left(0, t, H_{0, \Gamma_{1}}^{1}(\Omega)\right)^{\circ}}
\end{aligned}
$$

Lemma 11. For $T$ and $T_{h}$ solution of the problems $(\mathscr{P})$ and $\left(\mathscr{P}_{h}\right)$, respectively, one has

$$
\begin{aligned}
\| T- & T_{h} \|_{L^{2}\left(0, t, H_{0, \Gamma_{1}}^{1}(\Omega)\right)} \\
\lesssim & h^{\sigma}\left(\|T\|_{L^{2}\left(0, t, H^{1+\sigma}(\Omega)\right)}+\|T\|_{H^{1}\left(0, t, H^{\sigma}(\Omega)\right)}\right. \\
& \left.+\|C\|_{L^{2}\left(0, t, H^{1+\sigma}(\Omega)\right)}+\|C\|_{H^{1}\left(0, t, H^{\sigma}(\Omega)\right)}\right) \\
& +\left(\frac{2 \sqrt{10} \alpha \rho^{2} N N_{C}}{\delta_{2} \delta_{1} \lambda \eta}+\frac{\sqrt{5} N N_{T}}{\delta_{2} \delta_{1} \lambda}\right) \\
& \times\left\|\psi-\psi_{h}\right\|_{L^{2}\left(0, t, \stackrel{\circ}{W}^{1,4}(\Omega)\right)} .
\end{aligned}
$$


Proof. By using Lemmas 9 and 10, we have

$$
\begin{aligned}
& \left\|T-T_{h}\right\|_{L^{2}\left(0, t, H_{0, \Gamma_{1}}^{1}(\Omega)\right)} \\
& \lesssim h^{\sigma}\left(\|C\|_{H^{1}\left(0, t, H^{\sigma}(\Omega)\right)}+\|C\|_{L^{2}\left(0, t, H^{1+\sigma}(\Omega)\right)}\right. \\
& \left.\quad+\|T\|_{H^{1}\left(0, t, H^{\sigma}(\Omega)\right)}+\|T\|_{L^{2}\left(0, t, H^{1+\sigma}(\Omega)\right)}\right) \\
& +\left(\frac{2 \sqrt{10} \alpha \rho^{2} N N_{C}}{\delta_{1} \lambda \eta}+\frac{\sqrt{5} N N_{T}}{\delta_{1} \lambda}\right)\left\|\psi-\psi_{h}\right\|_{L^{2}\left(0, t, \stackrel{\leftrightarrow}{W}^{1,4}(\Omega)\right)} \\
& +\frac{2 \sqrt{5} \rho^{4} \alpha^{2} E N_{C}}{\delta_{1} \lambda \eta R T_{i}^{2}}\left\|T-T_{h}\right\|_{L^{2}\left(0, t, H_{0, \Gamma_{1}}^{1}(\Omega)\right)^{*}}
\end{aligned}
$$

If we set

$$
\delta_{2}=1-\frac{2 \sqrt{5} \rho^{4} \alpha^{2} E N_{C}}{\delta_{1} \lambda \eta R T_{i}^{2}}>0,
$$

then we get

$$
\begin{aligned}
& \delta_{2}\left\|T-T_{h}\right\|_{L^{2}\left(0, t, H_{0, \Gamma_{1}}^{1}(\Omega)\right)} \\
& \lesssim h^{\sigma}\left(\|T\|_{L^{2}\left(0, t, H^{1+\sigma}(\Omega)\right)}+\|T\|_{H^{1}\left(0, t, H^{\sigma}(\Omega)\right)}\right. \\
& \left.+\|C\|_{L^{2}\left(0, t, H^{1+\sigma}(\Omega)\right)}+\|C\|_{H^{1}\left(0, t, H^{\sigma}(\Omega)\right)}\right) \\
& \left.+\left(\frac{2 \sqrt{10} \alpha \rho^{2} N N_{C}}{\delta_{1} \lambda \eta}+\frac{\sqrt{5} N N_{T}}{\delta_{1} \lambda}\right) \times\left\|\psi-\psi_{h}\right\|_{L^{2}(0, t, W} \stackrel{\circ}{1,4}(\Omega)\right) .
\end{aligned}
$$

Lemma 12. One assumes that the hypotheses $\left(H_{1}\right),\left(H_{2}\right)$, and $\left(\mathrm{H}_{3}\right)$ are verified, so

$$
\begin{aligned}
&\left\|\omega-\omega_{h}\right\|_{L^{2}\left(0, t, L^{2}(\Omega)\right)} \\
& \lesssim h^{\sigma}\left(\|T\|_{L^{2}\left(0, t, H^{1+\sigma}(\Omega)\right)}+\|T\|_{H^{1}\left(0, t, H^{\sigma}(\Omega)\right)}\right. \\
&+\|C\|_{L^{2}\left(0, t, H^{1+\sigma}(\Omega)\right)}+\|C\|_{H^{1}\left(0, t, H^{\sigma}(\Omega)\right)} \\
&+\|\psi\|_{L^{2}\left(0, t, W^{1+\sigma, 4}(\Omega)\right)}+\|\omega\|_{H^{1}\left(0, t, H^{\sigma}(\Omega)\right)} \\
&\left.+\|\omega\|_{L^{2}\left(0, t, H^{\sigma}(\Omega)\right)}\right) .
\end{aligned}
$$

Proof. First of all, by using Lemmas 7, 8, and 11, we obtain

$$
\begin{aligned}
&\left\|\omega-\omega_{h}\right\|_{L^{2}\left(0, t, L^{2}(\Omega)\right)} \\
& \lesssim h^{\sigma}\left(\|T\|_{L^{2}\left(0, t, H^{1+\sigma}(\Omega)\right)}+\|T\|_{H^{1}\left(0, t, H^{\sigma}(\Omega)\right)}\right. \\
&+\|C\|_{L^{2}\left(0, t, H^{1+\sigma}(\Omega)\right)}+\|C\|_{H^{1}\left(0, t, H^{\sigma}(\Omega)\right)} \\
&+\|\omega\|_{L^{2}\left(0, t, H^{\sigma}(\Omega)\right)}+\|\omega\|_{H^{1}\left(0, t, H^{\sigma}(\Omega)\right)} \\
&\left.+\|\psi\|_{L^{2}\left(0, t, W^{1+\sigma, 4}(\Omega)\right)}\right) \\
&+\sqrt{3} \frac{\beta g}{\mu_{p}}\left(\frac{2 \sqrt{10} \alpha \rho^{2} N N_{C}}{\delta_{2} \delta_{1} \lambda \eta}+\frac{\sqrt{5} N N_{T}}{\delta_{2} \delta_{1} \lambda}\right) \\
& \times\left\|\omega-\omega_{h}\right\|_{L^{2}\left(0, t, L^{2}(\Omega)\right)}
\end{aligned}
$$

If we set

$$
A=\left(\frac{2 \sqrt{10} \alpha \rho^{2} N_{C}}{\eta}+\sqrt{5} N_{T}\right),
$$

assuming that

$$
\frac{\sqrt{3} \beta g N}{\delta_{2} \delta_{1} \lambda \mu_{p}} A \leq 1-\delta_{7} \quad \text { with } 0<\delta_{7}<1,
$$

for example, if $\delta_{7}=1 / 2$, we obtain

$$
\delta_{2} \delta_{1}>\frac{2 \sqrt{3} \beta g N}{\lambda \mu_{p}} A,
$$

and then we obtain

$$
\begin{aligned}
&\left\|\omega-\omega_{h}\right\|_{L^{2}\left(0, t, L^{2}(\Omega)\right)} \\
& \lesssim h^{\sigma}\left(\|T\|_{L^{2}\left(0, t, H^{1+\sigma}(\Omega)\right)}+\|T\|_{H^{1}\left(0, t, H^{\sigma}(\Omega)\right)}\right. \\
&+\|C\|_{L^{2}\left(0, t, H^{1+\sigma}(\Omega)\right)}+\|C\|_{H^{1}\left(0, t, H^{\sigma}(\Omega)\right)} \\
&+\|\omega\|_{L^{2}\left(0, t, H^{\sigma}(\Omega)\right)}+\|\omega\|_{H^{1}\left(0, t, H^{\sigma}(\Omega)\right)} \\
&\left.+\|\psi\|_{L^{2}\left(0, t, W^{1+\sigma, 4}(\Omega)\right)}\right) .
\end{aligned}
$$

Finally, using Lemmas $8,10,11$, and 12 , we obtain the a priori error estimate at the same time on the temperature, the concentration, the stream function, and the vorticity, so the main Theorem 1 is proved.

\section{Conclusion}

The propagation of reaction front in porous media is modelled by a system of equations, coupling hydrodynamic equations and the reaction-diffusion equations. We have taken into account Darcy-Boussinesq approximation. We have adopted the stream function-vorticity formulation of the Darcy equation and we have chosen the appropriate functional framework for our variational problem. We have proved the existence result for the semidiscrete solution. Furthermore, we have established an optimal a priori estimate on the temperature, the concentration, the stream function, and the vorticity.

\section{Conflict of Interests}

The authors declare that there is no conflict of interests regarding the publication of this paper.

\section{References}

[1] B. Jha, L. Cueto-Felgueroso, and R. Juanes, "Quantifying mixing in viscously unstable porous media flows," Physical Review E, vol. 84, no. 6, Article ID 066312, 2011.

[2] K. Vafai and P. C. Huang, "Analysis of heat transfer regulation and modification employing intermittently emplaced porous cavities," Journal of Heat Transfer, vol. 116, no. 3, pp. 604-613, 1994. 
[3] D. A. Nield and A. Bejan, Convection in Porous Media, Springer, New York, NY, USA, 2006.

[4] D. A. S. Rees, "The stability of Darcy-Bénard convection," Handbook of Porous Media, vol. 12, pp. 521-528, 2000.

[5] S. Rionero, "Global non-linear stability in double diffusive convection via hidden symmetries," International Journal of Non-Linear Mechanics, vol. 47, no. 1, pp. 61-66, 2012.

[6] F. Brezzi and M. Fortin, Mixed and Hybrid Finite Element Methods, Springer, New York, NY, USA, 1991.

[7] P. G. Ciarlet, The Finite Element Method for Elliptic Problems, North-Holland, Amsterdam, Netherlands, 1978.

[8] G. H. Meyer, Initial Value Methods for Boundary Value Problems, Academic Press, New York, NY, USA, 1973.

[9] C. G. Simader, On Dirichlet's Boundary Value Problem: LPTheory Based on Generalization Garding's Inequality, vol. 268, Springer, 1972. 


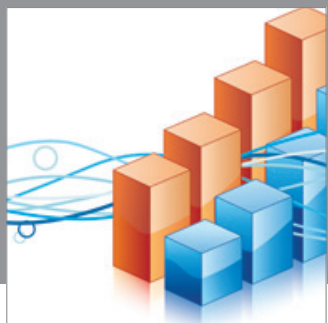

Advances in

Operations Research

mansans

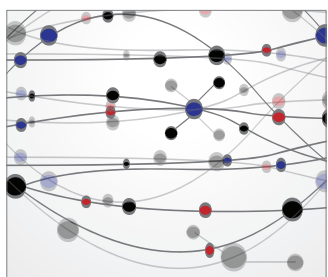

The Scientific World Journal
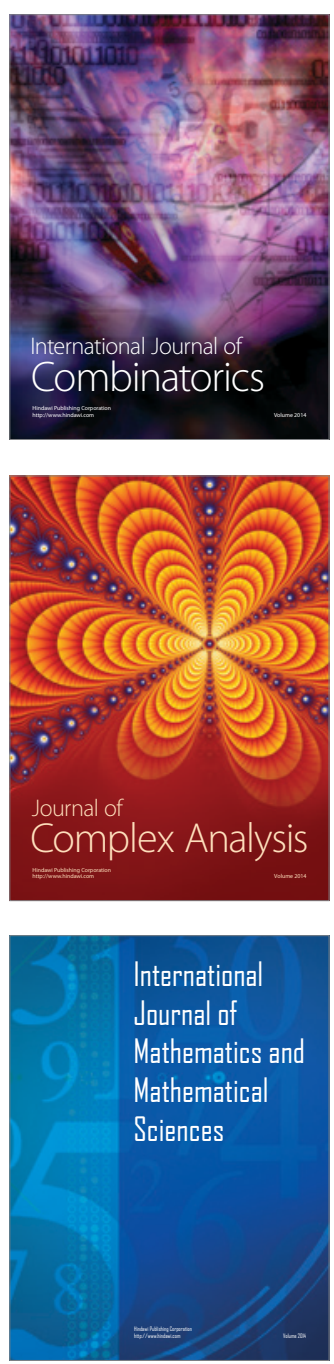
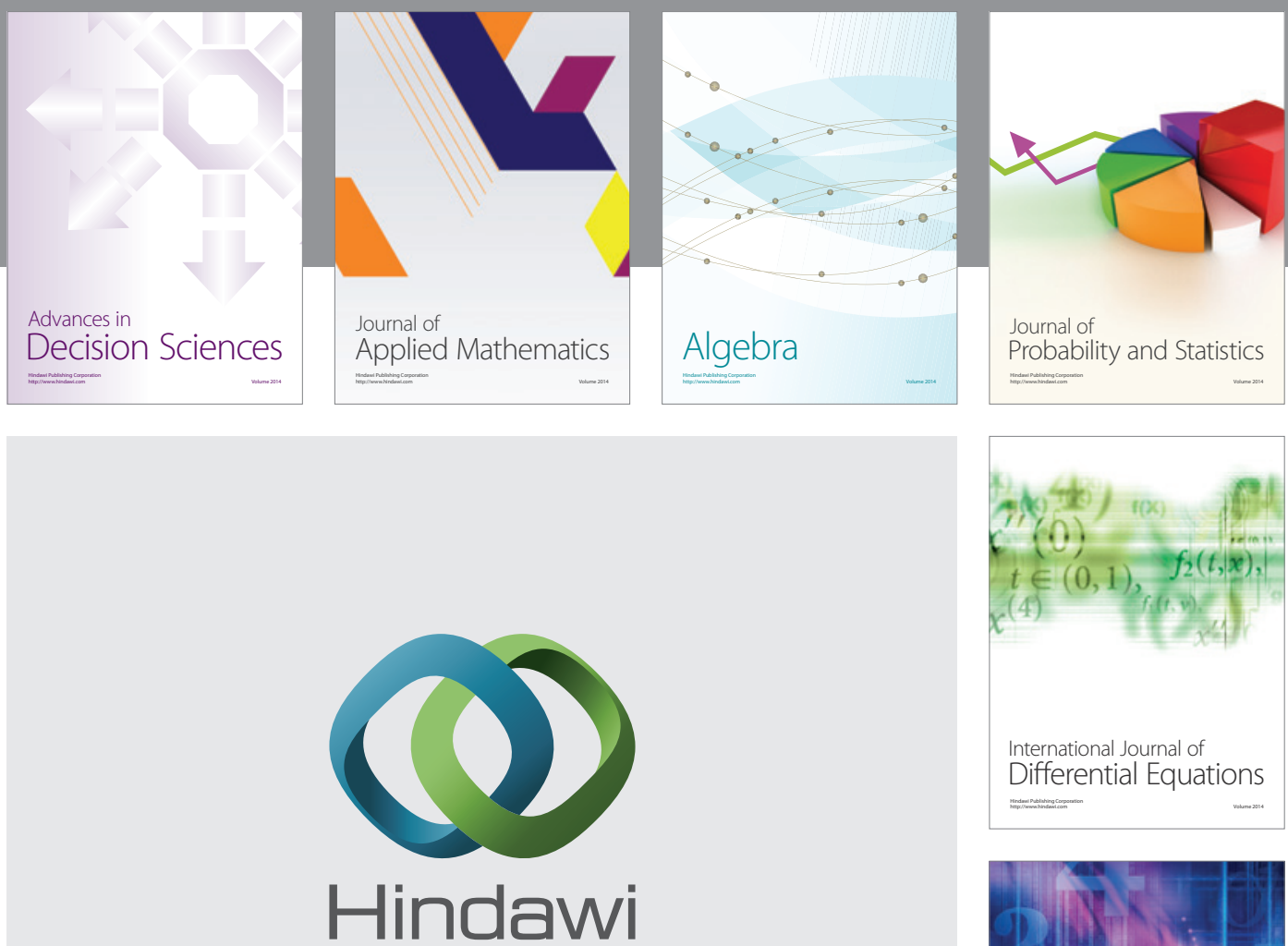

Submit your manuscripts at http://www.hindawi.com
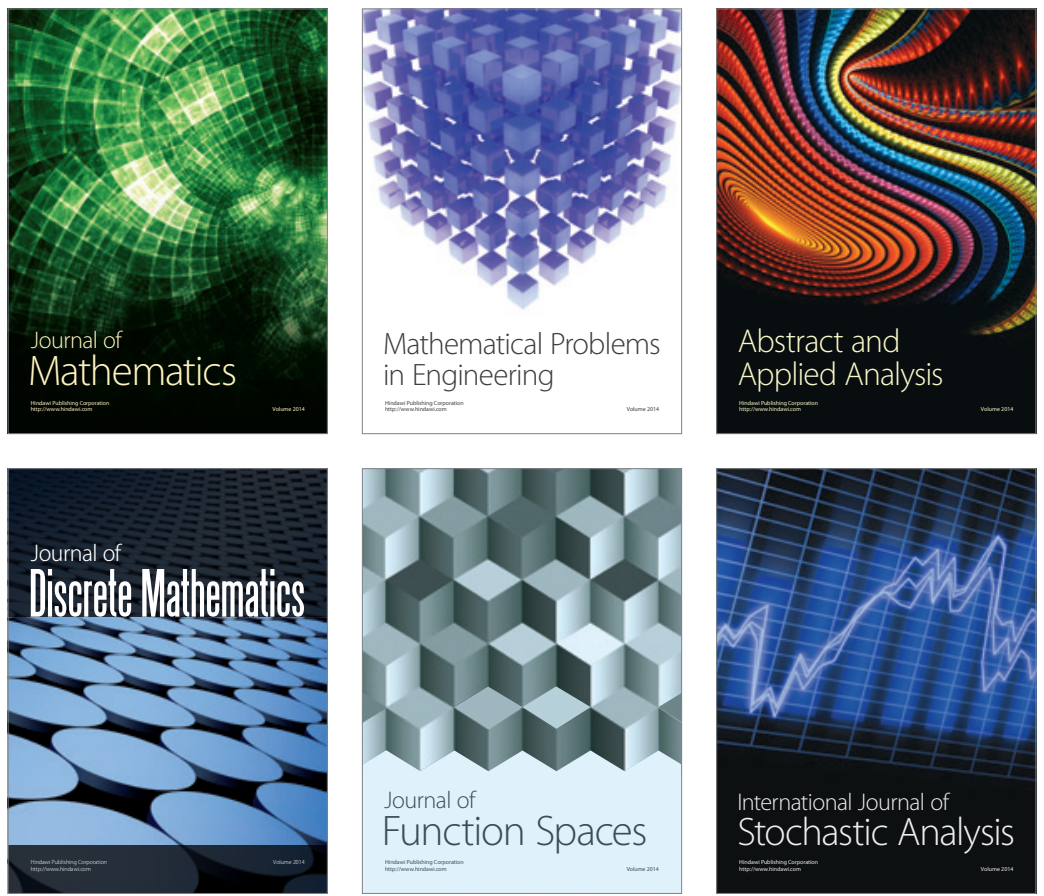

Journal of

Function Spaces

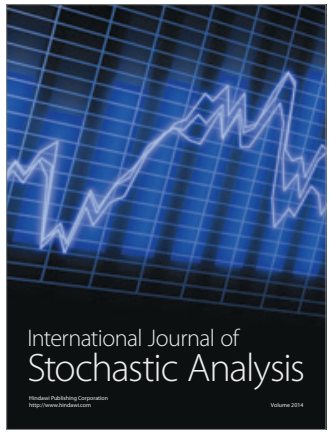

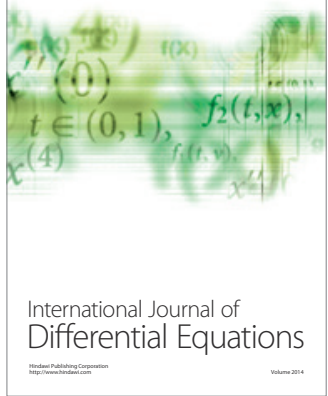
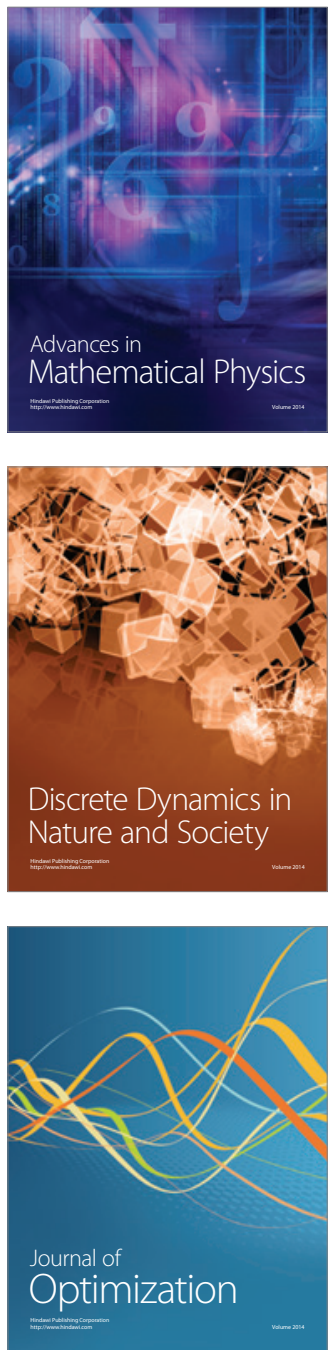